\title{
Risk of Dementia and Alcohol and Wine Consumption: a Review of Recent Results
}

\author{
LUC LETENNEUR
}

INSERM U593, Case11, Université Victor Segalen Bordeaux 2,146 rue Leo Saignat 33076 Bordeaux Cedex, France

\begin{abstract}
The term dementia refers to a clinical syndrome of acquired intellectual disturbances produced by brain dysfunction. Dementia may result from a wide variety of disorders, including degenerative (e.g. Alzheimer's disease, AD), vascular (e.g. multi-infarct dementia), and traumatic (e.g. head injury). Long-term abuse of alcohol is related to the development of the Wernicke-Korsakoff's syndrome or alcohol dementia. However, light to moderate alcohol intake might also reduce the risk of dementia and AD. In Bordeaux (France), a population-based prospective study found that subjects drinking 3 to 4 standard glasses of wine per day (> 250 and up to $500 \mathrm{ml}$ ), categorized as moderate drinkers, the crude odds ratio (OR) was 0.18 for incident dementia $(\mathrm{p}<0.01)$ and 0.25 for Alzheimer's disease $(\mathrm{p}<0.03)$, as compared to the non-drinkers. After adjusting for age, sex, education, occupation, baseline cognitive performances and other possible confounders, the ORs were respectively $0.19(\mathrm{p}<0.01)$ and $0.28(\mathrm{p}<0.05)$. In the 922 mild drinkers $(<1$ to 2 glasses per day) there was a negative association only with $\mathrm{AD}$, after adjustment $(\mathrm{OR}=0.55 ; \mathrm{p}<0.05)$. The inverse relationship between moderate wine drinking and incident dementia was explained neither by known predictors of dementia nor by medical, psychological or socio-familial factors. These results were confirmed from data of the Rotterdam study. Light-to-moderate drinking (one to three drinks per day) was significantly associated with a lower risk of any dementia (hazard ratio 0.58 [95\% CI 0.38-0.90]) and vascular dementia (hazard ratio 0.29 [0.09-0.93]). No evidence that the relation between alcohol and dementia varied by type of alcoholic beverage was found. Stroke constitutes one of the most common causes of serious functional impairment in developed countries. Ischaemic strokes represent about $80 \%$ of all strokes. Several studies have been published and the overall conclusion is that heavy drinking is a risk factor for most stroke subtypes. Regular light to moderate drinking seemed to be associated with a decreased risk for ischaemic stroke.
\end{abstract}

Key words: Alcohol, Alzheimer's disease, dementia, wine.

Abbreviations: AD: Alzheimer's disease; OR: odds ratio.

\section{INTRODUCTION}

Alcohol consumption is known for its psychotropic effects. On the nervous system, behavioral changes after alcohol intake vary according to the quantity of ethanol ingested: psycho-stimulation if the quantity is lower than $0.5 \mathrm{~g} / \mathrm{l}$, and sedation beyond that dose. Psycho-stimulation is associated with a relaxation of inhibitions: cognitive tasks are done more quickly, with a feeling of easiness but with an increased error rate. There is also a risk- taking attitude, especially when driving a car. Cognitive impairment is frequently observed in heavy drinkers and memory, visuomotor capacity or abstract thinking are affected. Excessive alcohol consumption is also responsible for Korsakoff's syndrome, also named alcoholic dementia. Actually, this disease is due to the lack of vitamin B1, frequently associated with malnutrition in heavy drinkers.

With the aging of the population, some pathologies such as dementia, become a public health problem. Dementia is characterized by the impairment of highly integrated cognitive functions including memory. The most frequent dementia is 
Alzheimer's disease, which is a degenerative dementia characterized by a progressive alteration of cognitive functions and represents $2 / 3$ of all dementia. Dementia frequency increases with age and varies from $1 \%$ in people aged 65 years, to more than 28 $\%$ in people aged more than 90 years (Lobo et al., 2000). The etiology is still unknown and the search for risk factors is still under way. Alcohol consumption is one of these risk factors, and has been studied in cohorts of subjects aged 65 years and older.

\section{PROSPECTIVE STUDIES}

In 1987, the PAQUID epidemiological research program was initiated to study normal and pathological brain aging (Letenneur et al., 1999). A cohort was constituted with 3777 elderly people living in 2 administrative areas in the southwest of France. Subjects were visited at home by a psychologist and several characteristics were collected, including wine consumption. Four categories were recorded: non-drinkers, mild drinkers (between 1 and 2 drinks per day), moderate drinkers ( 3 to 4 drinks per day), and heavy drinkers (more than 4 drinks per day). In this sample, wine was the main alcoholic beverage. A neuropsychological battery was administered in order to test cognitive performance of the subjects. Then, if the subjects had signs of dementia, a neurologist was appointed to make a diagnosis. The follow up of the sample was done 1 year, 3, 5, 8 and 10 years after the initial visit. At each visit, a questionnaire and the psychometric battery were administered, and the diagnosis of dementia was established. A first analysis (Orgogozo et al., 1997) showed that moderate wine consumption was associated with a lower risk of incident dementia 3 years after the initial visit (Table I). The risk of developing dementia was divided by 5 $(\mathrm{OR}=0.19 ; 95 \%$ CI $[0.05 ; 0.66])$ in moderate drinkers. When considering Alzheimer's disease, mild drinkers and moderate drinkers had a significant decrease in risk $(\mathrm{OR}=0.55 ; 95 \%$ CI [0.31; 0.99] and $\mathrm{OR}=0.28 ; 95 \%$ CI [0.08; 0.99], respectively). Another analysis (Lemeshow et al., 1998) confirmed these results after adjustment for many other potential confounding factors.

Other authors have recently published results on the association between alcohol consumption and the risk of incident dementia on cohorts of elderly subjects. In Sweden, 402 subjects aged 75 years and older at baseline were followed for 6 years and 179 developed a dementia. A reduction of the risk by two $(\mathrm{RR}=0.6 ; 95 \%$ CI $[0.40$; 1.0]) was also observed among mild to moderate drinkers compared to non drinkers (Huang et al., 2002).

TABLE I

Risk of incident dementia or Alzheimer's disease according to wine consumption

\begin{tabular}{|c|c|c|c|c|c|}
\hline \multirow[b]{2}{*}{ Wine consumption } & \multirow[b]{2}{*}{$\mathrm{n}$} & \multicolumn{2}{|c|}{ Dementia } & \multicolumn{2}{|c|}{ Alzheimer's disease } \\
\hline & & OR & $\begin{array}{l}95 \% \text { Confidence } \\
\text { Interval }\end{array}$ & OR & $\begin{array}{c}95 \% \text { Confidence } \\
\text { Interval }\end{array}$ \\
\hline Non drinker & 971 & 1 & Reference & 1 & Reference \\
\hline Mild drinker & 922 & 0.81 & $0.50-1.30$ & 0.55 & $0.31-0.99$ \\
\hline Moderate drinker & 318 & 0.19 & $0.05-0.66$ & 0.28 & $0.08-0.99$ \\
\hline Heavy drinker & 62 & 0.31 & $0.04-2.42$ & 0.48 & $0.06-3.92$ \\
\hline
\end{tabular}

Logistic regression adjusted for age, sex, educational level, occupation and initial cognitive performance. PAQUID, 1988-1991. 
Another result was obtained from the Rotterdam Study in the Netherlands (Ruitenberg et al., 2002). In 1990, 7983 subjects aged 55 years and older were included in a prospective study. After 6 years of follow up, 197 incident cases of dementia were identified. Alcohol consumption was determined at the initial visit. Subjects were divided into individuals who drank less than 1 drink of alcohol per week, more than 1 drink per week but less than 1 drink per day, 1 to 3 drinks per day, and more than 4 drinks per day. The type of alcoholic beverage (wine, beer, spirits) was also recorded. The analysis showed that the risk of developing dementia was significantly lower in people who drank 1 to 3 drinks per week $(\mathrm{OR}=0.58 ; 95 \% \mathrm{CI}$ $[0.38 ; 0.90])$. The risk was lower, but nonsignificantly, in mild drinkers $(\mathrm{OR}=0.75$; $95 \%$ CI $[0.51 ; 1.11])$ and in people who drank more than 4 drinks per day, the risk was equal to the risk of non drinkers $(\mathrm{OR}=$ $1 ; 95 \%$ CI $[0.39 ; 2.59])$. When vascular dementia was considered, a J shape curve was observed. Moreover, the association seemed to be modified according to genetic factors. Indeed, $\varepsilon 4$ allele of Apolipoprotein $\mathrm{E}$ is a risk factor for Alzheimer's disease. Among $\varepsilon 4$ carriers, and taking non-drinkers as the reference, the risk was estimated to be 0.71 (95 \% CI $[0.40 ; 1.25])$ in people who drank less than 1 drink per week, 0.46 (95\% CI $[0.25 ; 0.84])$ for the category $>1$ drink/week - < 1 drink/day, and $0.56(95 \%$ CI $[0.32 ; 1.00])$ in the category $1-3$ drinks/ day. Among non-carriers, the risks were respectively 1.03 (95\% CI [0. 60; 1.78]), 1.07 (95\% CI $[0.62 ; 1.85])$ and $0.53(95 \%$ CI $[0.27 ; 1.02])$. Therefore, a light to moderate alcohol consumption seemed to reduce the risk of developing Alzheimer's disease among those subjects with the highest risk.

The question about the type of alcoholic beverage has been studied, but no difference has been observed between wine, beer or spirits. However, another cohort study showed a difference according to the type of alcohol. In the Canadian Study of Health and Aging (Lyndsay et al., 2002), the follow up of 4088 for 5 years showed a reduction of the risk of developing dementia $(\mathrm{OR}=0.69$;
$95 \%$ CI $[0.47 ; 1.00])$ in alcohol drinkers compared to non drinkers. In addition, the risk was lower in wine drinkers $(\mathrm{OR}=0.49$; $95 \%$ CI $[0.28 ; 0.88])$ than in beer or spirit drinkers $(\mathrm{OR}=0.84 ; 95 \% \mathrm{CI}[0.51 ; 1.41])$ and $\mathrm{OR}=0.78 ; 95 \% \mathrm{CI}[0.52 ; 1.19])$, respectively.

In another cohort (the Copenhagen City Heart Study, Truelsen et al., 2002), the alcohol consumption recorded in 1976 in a cohort initially designed to study cardiovascular diseases has been linked to the risk of having a dementia in 1990-94. Compared to non drinkers, the risk of developing dementia was significantly lower among occasional wine drinkers $(\mathrm{OR}=0.43 ; 95 \%$ CI $[0.23 ; 0.82])$, in weekly drinkers $(\mathrm{OR}=0.33 ; 95 \% \mathrm{CI}$ $[0.13 ; 0.86])$, but non significant in daily drinkers $(\mathrm{OR}=0.57 ; 95 \% \mathrm{CI}[0.15 ; 2.11])$. However, an increased risk was observed for beer $(\mathrm{OR}=2.28 ; 95 \%$ CI $[1.13 ; 4.60]$ in occasional drinkers, $\mathrm{OR}=2.15 ; 95 \% \mathrm{CI}$ $[0.98 ; 4.78]$ in weekly drinkers and $\mathrm{OR}=1.73 ; 95 \%$ CI $[0.75 ; 3.99]$ in daily drinkers) or for spirits $(\mathrm{OR}=0.81 ; 95 \%$ CI $[0.42 ; 1.57]$ in occasional drinkers, $\mathrm{OR}=1.65 ; 95 \% \mathrm{CI}[0.74 ; 3.69]$ in weekly drinkers and $\mathrm{OR}=1.12 ; 95 \% \mathrm{CI}$ $[0.43 ; 2.92]$ in daily drinkers).

\section{DISCUSSION}

These prospective studies all lead to the same conclusion, i.e. moderate alcohol consumption is associated with a reduction of the risk of developing dementia. However, these are observational studies, and it is not a proof of a causal association. Indeed, a specific selection of the sample may explain such results. That is why in most studies, the analysis of mortality has been performed in order to find whether premature death due to alcohol consumption would alter the association between alcohol and dementia. Actually, in these samples and for the age groups considered, mortality was lower among drinkers. Therefore, the results cannot be explained by differential mortality of drinkers. Other factors may explain the differences between drinkers and non- 
drinkers. Most studies have adjusted for many potential confounding factors (age, sex, education, marital status, income, etc.) which may be associated to the disease and alcohol consumption. Again, the association was not modified by these adjustments, but other factors not considered in the analyses may explain these results. Indeed, moderate drinkers may be moderate for many others risk factors that may lead to a decreased risk of developing dementia, independently from alcohol consumption. Are these subjects exposed to specific risk factors, specific diets, or have a better social network? These questions remain without answer. However, in a domain where behavior is so profoundly influenced by cultural and social environments, it seems very unwise to consider alcohol as the only factor.

Which mechanism could be involved in the risk reduction of developing a dementia or Alzheimer's disease? Several hypotheses have been given, but none has been confirmed. A first hypothesis is the reduction of the cardiovascular risk factors, either by inhibiting platelet aggregation, or by the modification of the lipid profile. Actually, the differential effect of alcohol in ApoE4 carriers could be explained by an increase of HDL cholesterol in these subjects who have a low level of HDL cholesterol and a high level of LDL cholesterol. Another possibility is the fact that alcohol might have a direct effect on cognition through release of acetylcholine in the hippocampus. Acetylcholine is involved in learning and memory mechanisms that are impaired in Alzheimer's disease.

Finally, between alcohol or wine, which one protects the most? For the moment, it is not possible to conclude from the available data. The main element favoring wine lies in its flavonoid content and its antioxidant activity. Commenges et al. (2000) have shown that a diet rich in flavonoids was associated with a lower risk of dementia. In addition, the association with wine consumption disappeared after controlling for diet and may indicate that wine is a marker of a healthier diet. Indeed, a Danish study showed that wine drinkers had a healthier diet than beer or spirit drinkers (Tjonneland et al., 1998). They ate more fruits and vegetables, more fish and used more often olive oil for cooking. This confirms the importance of considering other factors before drawing conclusions.

In conclusion, should we advise to drink alcohol moderately to avoid the development of some diseases? Epidemiological studies have shown that possible beneficial effects of alcohol consumption on health were apparent only after age 40 in men and age 50 in women. Before that age, the consequences of alcohol consumption are more negative in terms of accidents, diseases directly linked to alcohol (cirrhosis, liver, mouth, larynx cancers, etc.) and the risk of alcohol addiction. The premature mortality attributable to alcohol (excluding accidents and injuries) is estimated to $15 \%$. After age 50 , and particularly in elderly whose occupational activity has stopped, a moderate alcohol consumption should not be prohibited. However, it seems premature to advise people who do not drink to start drinking, since the risk of addiction is far from being negligible (it is estimated to be $13 \%$ in mature adults). Alcohol consumption should remain a pleasure and should not be considered as a medication.

\section{REFERENCES}

COMMENGES D, SCOTET V, RENAUD S, JACQMINGADDA H, BARBERGER-GATEAU P, DARTIGUES JF (2000) Intake of flavonoids and risk of dementia. Eur J Epidemiol 16:357-363

HUANG W, QIU C, WINBLAD B, FRATIGLIONI L (2002) Alcohol consumption and incidence of dementia in a community sample age 75 years and older. J Clin Epidemiol 55:959-64

LEMESHOW S, LETENNEUR L, DARTIGUES JF, LAFONT S, ORGOGOZO JM, COMMENGES D (1998) An illustration of analysis taking into account complex survey considerations: the association between wine consumption and dementia in the Paquid study. Am J Epidemiol 148: 298-306

LETENNEUR L, GILLERON V, COMMENGES D, HELMER C, ORGOGOZO JM, DARTIGUES JF (1999) Are sex and educational level independent predictors of dementia and Alzheimer's disease? Incidence data from the PAQUID project. J Neurol Neurosurg Psychiatry 66:177-183

LINDSAY J, LAURIN D, VERREAULT R, HEBERT R, HELLIWELL B, HILL G, Mc DOWELL I (2002) Risk factors for Alzheimer's disease: A prospective analysis of the Canadian Study of Health and Aging. Am J Epidemiol 156:445-53 
LOBO A, LAUNER LJ, FRATIGLIONI L, AANDERSEN K, DI CARLO A, BRETELER MM, COPELAND JR, DARTIGUES JF, JAGGER C, MARTÍNEZ-LAGE J, SOININEN H, HOFMAN A (2000) Prevalence of dementia and major subtypes in Europe: A collaborative study of population-based cohorts. Neurologic Diseases in the Elderly Research Group. Neurology 54 (Suppl 5):S4-9

ORGOGOZO JM, DARTIGUES JF, LAFONT S, LETENNEUR L, COMMENGES D, SALAMON R, RENAUD S, BRETELER M (1997) Wine consumption and dementia in the elderly: a prospective community study in the Bordeaux area. Rev Neurol 153: 3: 185-192
RUITENBERG A, VAN SWIETEN J, WITTEMAN J, MEHTA K, VAN DUIJN C, HOFMAN A, BRETELER M (2002) Alcohol consumption and risk of dementia: the Rotterdam study. Lancet 359:281-286

TJONNELAND A, GRONBAEK M, STRIPP C, OVERVAD K (1998) Wine intake and diet in a random sample of 48,763 Danish men and women. Am J Clin Nutrition 69:46-54

TRUELSEN T, THUDIUM D, GRONBAEK M (2002) Amount and type of Alcohol and risk of dementia. The Copenhagen Heart Study. Neurology 59:1313-1319 